\title{
Self-organized Nanogels of Polysaccharide Derivatives in Anti-Cancer Drug Delivery
}

\author{
Sin-jung Park and Kun $\mathrm{Na}^{\dagger}$ \\ Department of Biotechnology, The Catholic University of Korea, 43-1 Yeokkok2-dong, Wonmi-gu, \\ Bucheon-si, Gyeonggi-do, 420-743, Korea \\ (Received July 20, 2010 • Revised July 30, 2010 • Accepted July 31, 2010)
}

\begin{abstract}
Self-organized nanogels from polysaccharide derivatives offer a promising approach in treatment of cancer due to their flexibility in chemistry and their ability to improve the therapeutic index of a drug by modifying biodistribution by their preferential localization at target sites and lower distribution in normal healthy tissues. These properties have promoted studies of active cancer targeting by self-organized nanogels for even better accumulation in solid tumors. However although many researchers have reported their potential by using cell culture systems and small animal tumor models in cancer therapy, these nanogels need more decoration such as conjugation with targeting moiety and endowment of stimuli-sensitivity for precise targeting of the cancer site. In this review, we summarize the recent efforts in developing novel targeting approaches via active endocytosis and stimuli-sensitive systems responding to hyperthermic or acidic tumor $\mathrm{pH}$ conditions.
\end{abstract}

Key words - Self-organized nanogels, Polysaccharide derivatives, Active targeting, Switching system

Over the past several decades, there have been advances in using of polymeric nanoparticles for drug delivery system. Polymeric nanoparticles are defined as particulate dispersions with a size in the range of 10-1000 nm (Mohanraj and Chen, 2007). It is divided to polymeric micelles and nanogels. Typical polymeric micelle is formed by a spontaneous self-assembly of individual polymeric molecules that are synthetic amphiphilic di-block or tri-block copolymers consist of hydrophilic and hydrophobic blocks. The micelles have diameters ranging from 10 to $100 \mathrm{~nm}$ and spherically shaped core-shell structures with a hydrophobic core and hydrophilic shell (Letchford and Burt, 2007; Rapoport, 2007). The hydrophobic core involves the pharmacological activities such as drug loading and release behavior, and hydrophilic shell affects the micelle's biodistribution and pharmacokinetic behavior.

Self-organized nanogels are formed by physically or chemically cross-linked polymer networks. Thus, it possesses multihydrophobic cores and hydrophilic area inner the gel (Fig. 1). The nanogels offer great advantage for polymer based drug delivery systems because of their high stability, loading capacity, novel functionality for further bioconjugation, controlled particle size, and biodegradability. Therefore, many groups have been studied as carriers for the transport of anticancer drug, protein, nucleic acid, and bioactive molecules (Oh et al.,

†Corresponding Author:

Tel : +82-2-2164-4832, E-mail : kna6997@catholic.ac.kr

DOI : 10.4333/KPS.2010.40.4.201
2008). The polymers with the property of physically crosslinking induced by hydrophobic or electrostatic interactions and/or hydrogen bonding with each other were more investigated to produce various nanogels due to its higher biocompatibility (Kabanov and Vinogradov, 2009).

For preparation of nanosystems, various polymers such as poly(lactic acid), poly(glycolic acid), polycaprolactone, polysaccharides, poly(acrylic acid) family, proteins or polypeptides (such as gelatin) have been employed (Liu et al., 2008). Among them, polysaccharides are the one of the most useful material for drug delivery because of their unique properties of biocompatibility, biodegradability, and abundance. A recent study concluded that polysaccharide based self-organized nanogels with targeting moieties or stimuli-sensitive properties are expected to provide the most efficacious delivery strategy (Benhar et al., 1994; Brigger et al., 2002; Nishiyama and Kataoka, 2006; Nobs et al., 2004). Improved performance against disease sites and minimized toxicity for healthy tissues seem to offer solutions to the problems associated with traditional chemotherapy (Balthasar et al., 2005; Khaw et al., 1991; Wong et al., 1997).

In this review, the major examples of polysaccharide based self-organized nanogels with targeting moieties or stimulussensitive properties that may be useful in tumor therapy are discussed in order to highlight the possible applications of such systems. The first major concern with these systems is the cancer specific-targeting strategy of the anticancer drug using selforganized nanogels with targeting moieties. The second con- 
(a)

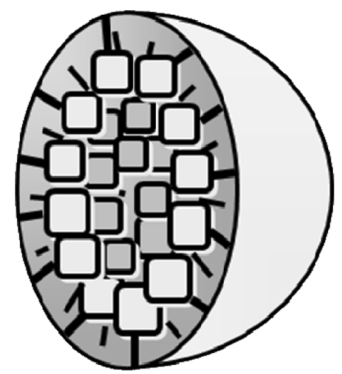

(b)

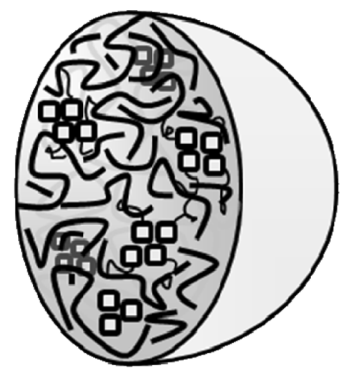

Figure 1. Schematic structures of (a) polymeric micelles and (b) self-assembled nanogels.

cern is controlling the anticancer drug release behavior in the polysaccharide based self-organized nanogels according to stimulus signals such as temperature and $\mathrm{pH}$.

\section{Polysaccharide Based Self-organized Nanogels}

For the past few decades, self-organized nanogels have been widely used to deliver anticancer drugs, proteins and genes. To enhance their therapeutic effects while minimizing their side effects, various polymers have been utilized in the preparation of self-organized nanogels. Among the polymeric materials, polysaccharides have a large potential in biomedical applications mainly due to it being a natural biomaterial, very stable, safe, non-toxic, hydrophilic, biocompatible, and biodegradable. Polysaccharides of algae, plant, microbial, and animal origin have been used in the field of biomaterial and drug delivery systems owing to their biocompatible and biodegradable properties. They have a broad range of molecular weight (MW), a lot of functional groups, and varying chemical compositions. Also, polysaccharides can be easily modified chemically and biochemically due to their various derivable functional groups. Because of all these merits, they are a promising material to be used in preparation of self-organized nanogels for anti-cancer drug delivery. In the following section, we mention several polysaccharides which are employed in the preparation of self-organized nanogels.

\section{Chitosan}

Chitosan (ChS) is a naturally occurring carbohydrate based biopolymer and it composed of 2-amino-deoxy-D-glucan combined with glycosidic $(\beta, 1-4)$ linkages. $\mathrm{ChS}$ is obtained by the deacetylation of chitin which is the major component of shells, crabs, and shrimp (Kumar et al., 2004). ChS has various merits such as biodegradability, biocompatibility, high positively charge density. Due to these properties, $\mathrm{ChS}$ can easily interact with negatively charged polymers and hence it has been used as gene delivery carriers. Also, $\mathrm{ChS}$ has been used for various

anti-cancer drug, protein delivery carriers (Oh et al., 2008). Park et al. reported on the effect of polymer molecular weight on tumor targeting characteristics (Park et al., 2007). They designed cholanic acid-conjugated glycol $\mathrm{ChS}$ with different molecular weights to enhance the in vivo tumor targeting. More recently, Hwang et al. reported that docetaxel-loaded hydrophobically modified glycol ChS (DTX-HGC) had a prolonged circulation time and tumor targeting ability, in tumorbearing mice (Hwang et al., 2008). In their previous report, they determined that the high molecular weight glycol ChS nanoparticles circulate in the blood for long periods and shown high tumor selectivity. On the basis of their previous study, they formed self-assembled doxetaxel-loaded HGC nanoparticles (DTX-HGC nanoparticles) optimized for high tumor targeting ability. The DTX-HGC nanoparticles formed selfassembled aggregates with a mean size of $350 \mathrm{~nm}$ as confirmed by dynamic light scattering and it was maintained for a 2 week period at $37^{\circ} \mathrm{C}$ in PBS. In vitro stability of DTX-HGC nanogels are examined by using a Sodium Dodecyl Sulfate Polyacrylamide Gel Electrophoresis (SDS-PAGE) in the presence of bovine serum albumin. As a result, these DTX-HGC nanogels maintained their stability in presence of excess serum. To estimate tumor-homing ability of the DTX-HGC nanogels the tumor-bearing mice were used, and it was monitored by whole-body, real-time NIR fluorescence intensity. The fluorescence of the whole animal was shown within $1 \mathrm{hr}$ of administration of Cy5.5-labeled DTX-HGC nanogels, and the subcutaneous tumor tissue steadily disappeared from the surrounding background tissue between $1 \mathrm{~h}$ to $72 \mathrm{~h}$ post-injection, with maximum contrast occurring at $12 \mathrm{~h}$ post-injection. In addition, after $12 \mathrm{~h}$ post-DTX-HGC nanoparticles injection in the tumor, NIR fluorescence intensity was observed strongly, while in the normal organs, the uptake of DTX-HGC nanoparticles was not significant. This result suggests that DTX-HGC nanogels have a great tumor-homing efficacy in vivo as compared to normal tissues. To determine the antitumor efficacy of DTX-HGC nanogels, tumor-bearing mice were used. Tumor volume was recorded in each tumor-bearing mouse after injection via the lateral tail vein with one of the following treatments; saline, HGC nanogels, free DTX, DTXHGC nanogels. There were no significant differences in size of the tumors until 10 days of treatment administration. However, after 28 days of treatment administration, tumor volume was conspicuously decreased in the group treated with DTX-HGC nanogels, as compared to the group treated with free DTX. All these results suggested that this high antitumor efficacy of DTX-HGC nanoparticles resulted from their high tumor targeting efficacy, and the sustained release profile of DTX from 
HGC nanoparticles within the tumor tissue.

\section{Pullulan}

Pullulan(PUL) is a linear polysaccharide with an $\alpha-(1-6)-$ linked maltotriosyl repeating unit. The merits of pullulan are that it is biocompatible, biodegradable, blood compatible, and non-immunogenic (Leathers, 2003; Rekha and Sharma, 2007). It can be chemically modified to various derivatives to endow amphiphilic property (Jeong et al., 2006; Jeong et al., 1999; Jung et al., 2003; Shen et al., 2008; Yang et al., 2008). Another advantage of PUL is that it binds strongly to asialoglycoprotein receptor and is internalized via receptor-mediated endocytosis (Kaneo et al., 2001; Tanaka et al., 2004). Therefore, a study on biomedical application of liver targeting through PUL has been developed. $\mathrm{Xi}$ at al. reported liver targeting of interferon through PUL conjugation (Xi et al., 1996). They chemically conjugated interferon (IFN) with PUL targeting to the liver. They examined body distribution of IFN-PUL conjugate in mice, by ${ }^{125}$ I-labeled IFN conjugated PUL. As per the report, in the mice, liver accumulation of IFN by conjugation with PUL strongly increased.

$\mathrm{Na}$ et al. reported on self-organized pullulan/deoxycholic acid nanogels (PUL/DOCA) (Na et al., 2006). In this study, PUL/DOCA was synthesized to prepare a self-organized nanogel in order to deliver an anticancer drug. As a hydrophobic moiety, DOCA, which is a bile acid, was used to prepare selfassembled nanogels to facilitate the oral administration of hydrophilic polymers. Three types of PUL/DOCA conjugates were prepared differing in the number of DOCA substitutions (DSs) and were named DS; 5, 8 and 11. The PUL/DOCA nanogels were less than $200 \mathrm{~nm}$ in size, and as DS of nanogels increased, the diameter of nanogels decreased. These results indicate that a high concentration of DOCA moiety in the PUL increases the chances of occurence of hydrophobic interactions and results in aggregation of particles. To determine the drugrelease behavior of these nanogels, doxorubicin (DOX) was loaded in the PUL/DOCA conjugates by using a dialysis method. Consequently, drug loading efficiency and loading content of PUL/DOCA increased as DS increased, while under the same circumstances, the particle size of the nanogels decreased. In PBS buffer, the DOX release behavior of DOXloaded PUL/DOCA nanogels was determined. The total amount of drug released from DS; 5, 8, and 11 in $24 \mathrm{~h}$ was 61 , 46 , and $38 \%$ respectively. The drug release rate from the nanogels containing more drug was slower than the release rate from the nanogels containing less drug. As a result, they suggested that the drug-release kinetics can be controlled by regulating the drug-loading content of the nanogels or by changing the DS. More recently, Oh et al. reported on preparation and characterization of stearic acid-PUL nanoparticles (Kim et al., 2008). They designed stearic acid conjugated PUL (SAP) as an anti-cancer drug delivery carrier. In their study, all-trans-retinoic acid (ATRA) was loaded in SAP, and these nanoparticles were maintained over a 5 week period.

\section{Hyaluronic Acid}

Hyaluronic acid (HA) is a glycosaminoglycan (GAG) and is one of the major components of extracellular matrix. It consists of repeating disaccharide units, $(\beta, 1-4)$-D-glucuronic acid (GlcUA), and ( $\beta, 1-3)-\mathrm{N}$-acetyl-D-glucosamine (GlcNAc) (Laurent, 1998). HA selectively interacted with HA-binding receptor such as Cluster determinant 44 (CD44) (Aruffo et al., 1990), receptor for HA acid-mediated motility (RAHMM) (Lugli et al., 2006) and lymphatic vessel endothelial receptor1 (LYVE-1) (Banerji et al., 1999) is over expressed on tumor cells (Ward et al., 2002). Thus, the application of HA leads to
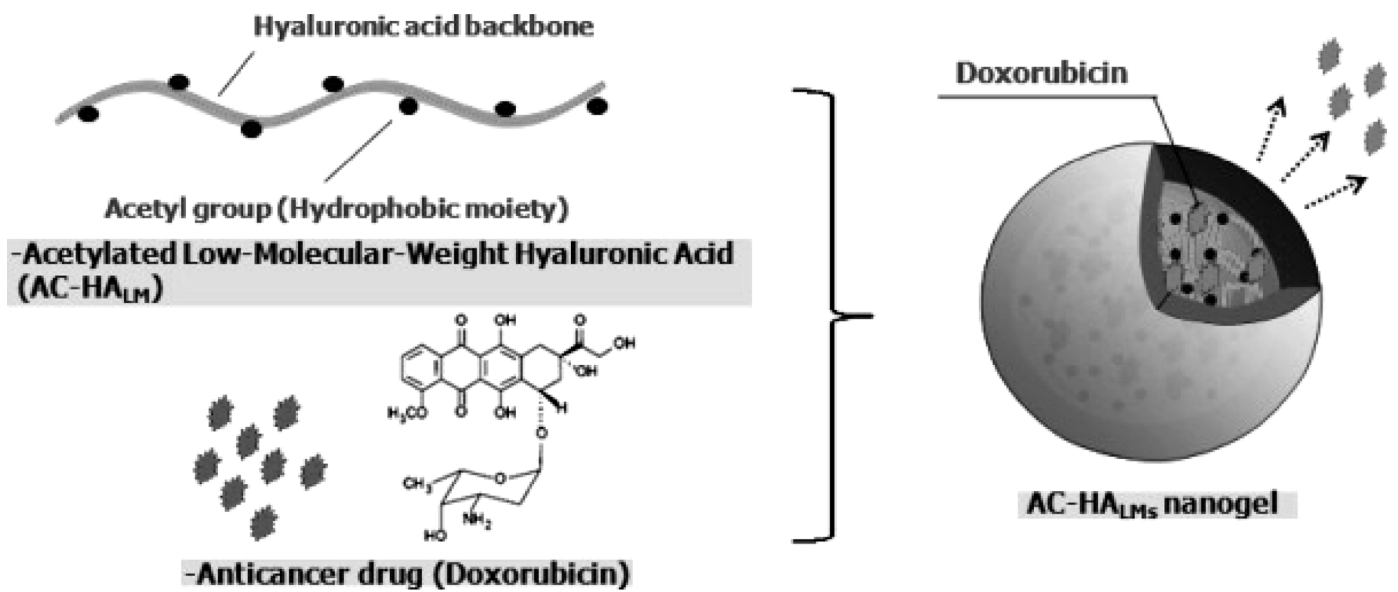

Figure 2. Schematic structure of self-organized nanogels with low molecular hyaluronic acid acetate $\left(\mathrm{AC}-\mathrm{HA} \mathrm{A}_{\mathrm{LM}}\right)$ in an aqueous medium. (reproduced from (Park et al., 2010) with permission from the publisher) 
enhance the selectivity of cancer and highly effective anti-cancer carriers. In a recent study, HA has been conjugated to different an anti-cancer drug or to different functional moieties. Drug conjugated HA derivative was studied in many research groups (Luo and Prestwich, 1999; Luo et al., 2000). In a more recent study, Homma et al. reported on synthesis and optimization of HA-methotrexate conjugates to maximize benefit in the treatment of osteoarthritis (Homma et al., 2009). Also, Xin studied the use of amino acid linkers in the conjugation of paclitaxel with HA as drug delivery system (Xin et al., 2010). In this study, they developed HA-paclitaxel conjugates using amino acids as linkers, and HA-amino acid-paclitaxel conjugate offered promising potential for further investigations in biomedical applications.

Choi et al. reported on self-assembled HA nanoparticles for active tumor targeting. They synthesized HA- $5 \beta$-cholanic acid conjugates (HA-CA conjugates) (Choi et al., 2010). In this study, they demonstrated that HA-CA conjugates have a strong HA receptor-binding affinity and, thus selectively target tumor tissue. Park et al. reported that self-organized nanogels were prepared from acetylated HA (AC-HA) (Park et al., 2010) (Fig. 2). AC-HA nanogels with different degrees of acetylation have hydrophobic cores from self-organizing acetyl groups that could affect pharmacokinetic properties such as drug loading and release behavior. Doxorubicine (DOX)-loaded AC-HA nanogels were internalized in cancer cells but were less internalized in normal cells. Such results indicate that AC-HA nanogels possesses cancer cell selectivity by HA receptor-binding.

\section{Chondroitin Sulfate}

Chondroitin sulfate (CS) is a sulfated glycosaminoglycan (GAG) composed of a chain of alternating sugars ( $N$-acetylgalactosamine and glucuronic acid). It is usually found attached to proteins as a part of proteoglycan (Mucci et al., 2000). CS covalently binds to endogenous proteins such as growth factors, and adhesion molecules. Moreover, CS binding receptor is overexpressed on cancer cells (Henke et al., 1996).

Recently, Park et al. reported that hydrophobically modified CS by using acetic anhydride was used as an anti-cancer drug carrier (Park et al., 2010). They designed hydrophobically modified CS (acetylated CS, AC-CS), and doxorubicin-loaded in AC-CS. In their research, AC-CS-DOX was physically stable and it maintained over a 3 week period. Also, AC-CS nanogels were internalized into tumor cells by HA receptor mediated endocytosis. The results suggest that AC-CS selforganized nanogels have great potential as effective anti-cancer drug carriers.

\section{Self-organized Nanogels with Targeting Moieties}

The tumor targeting approach in cancer chemotherapy is thought to provide a potential advantage of target site-specific delivery of anti-cancer drugs. For tumor targeting purposes, tumor cell-specific or over-expressed markers such as antigens and receptors have been employed (Benhar et al., 1994; Brigger et al., 2002; Nishiyama and Kataoka, 2006; Torchilin, 2006). The tumor targeting moieties are conjugated to drugcarrier system through covalent or non-covalent binding (Jaracz et al., 2005; Nobs et al., 2004). These tumor targeting moieties have strongly effect on in vivo system owing to the enhanced permeability retention (EPR) effect (Balthasar et al., 2005; Khaw et al., 1991; Wong et al., 1997) of nanocarriers and the tumor specific recognition activity. In this part, polysaccharide based self-organized nanogels decorated with tumor cell targeting moiety such as vitamins (folic acid, vitamin $H$, vitamin $B_{12}$, etc,.), and antibody (Bagalkot et al., 2006). All living cells require vitamins for survival, most of the tumor cells consume vitamins at a higher rate than normal cells in order to divide rapidly which requires receptors overexpressed in the tumor cells. Therefore, vitamin-decorated drug carriers are attractive to the tumor cells. Recently, molecules essential for tumor growth have been used in anti-cancer drug delivery systems (Bae and Kataoka, 2006; Lu and Low, 2002).

Vitamin $\mathrm{B}_{12}$ is another member of the vitamin $\mathrm{B}$ complex (RIcKms et al., 1948; Seetharam et al., 1999). Vitamin $B_{12}$ is an important factor for maintaining differentiation, proliferation, and metabolic status of cells (Bougrine et al., 1996; Seetharam and Li, 2000). It was generally known that vitamin $\mathrm{B}_{12}$ binds to plasma transcobalamin II (TC II) and is uptaken in all cells via a specific receptor called TC II receptor (TC II-R) by receptor-mediated endocytosis. The fact that vitamin $B_{12}$ is accumulated in tumors in rats (Flodh and Ullberg, 1968) and humans (Collins et al., 2000) has been identified by radiolabeled vitamin $\mathrm{B}_{12}$ analogues used to detect occult tumors.. In

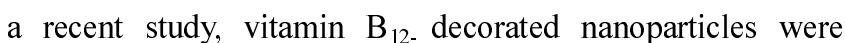
developed to deliver anti-cancer drug to the tumor site.

Folic acid (FA) has been studied as a promising targeting ligand for cancer detection in ovarian, breast, lung, kidney and brain tumors that over-express folate binding proteins. Folate receptor (FR) is overexpressed in about $20-50 \%$ of solid tumors originating from kidney, lung, breast, bladder, and pancreas (Normanno et al., 2005). Receptor-mediated endocytosis occurs via a non-lysosomal route and the delivery of anticancer drugs by loading, conjugating and complexing using folate decorated carriers has been achieved via this mechanism. 
Kim et al. reported on minimalism in fabrication of selforganized nanogels holding both anti-cancer drug and targeting moiety (Kim et al., 2008). To target cancer cells, FA was directly coupled with PUL backbone by ester linkage (FA/PUL conjugate).

Huang et al. reported on folate-mediated CS -Pluronic 127 nanogels as a drug carrier (Huang et al., 2009). To synthesize CS-decorated Pluronic 127 (CS-PF127), the hydroxyl groups of Pluronic were acrylated to react with methacrylated CS. The further grafting of FA moiety onto CS-PF127 was prepared in order to achieve tumor-targeting. Pluronic 127 has been used to promote active membrane transport of numerous anticancer drugs because of the Pluronic effects in multi drug resistance cells (MDR) which can be attributed to be due to inhibition of drug efflux transporters. In their study, they reported that folic acid conjugated CS-PF127 (FA-CS-PF127) was internalized by $\mathrm{CS}$ receptor-mediated and synergetic cellular uptake by FAbinding receptors. A competitive inhibition study between FACS-PF127 nanogels and FA-CS-PF127 with free FA was tested by flow cytometry (Fig. 3). KB cells were incubated with free FA for $30 \mathrm{~min}$ before adding FA-CS-PF127. Free FA was found to bind to folate receptors on $\mathrm{KB}$ cell membranes
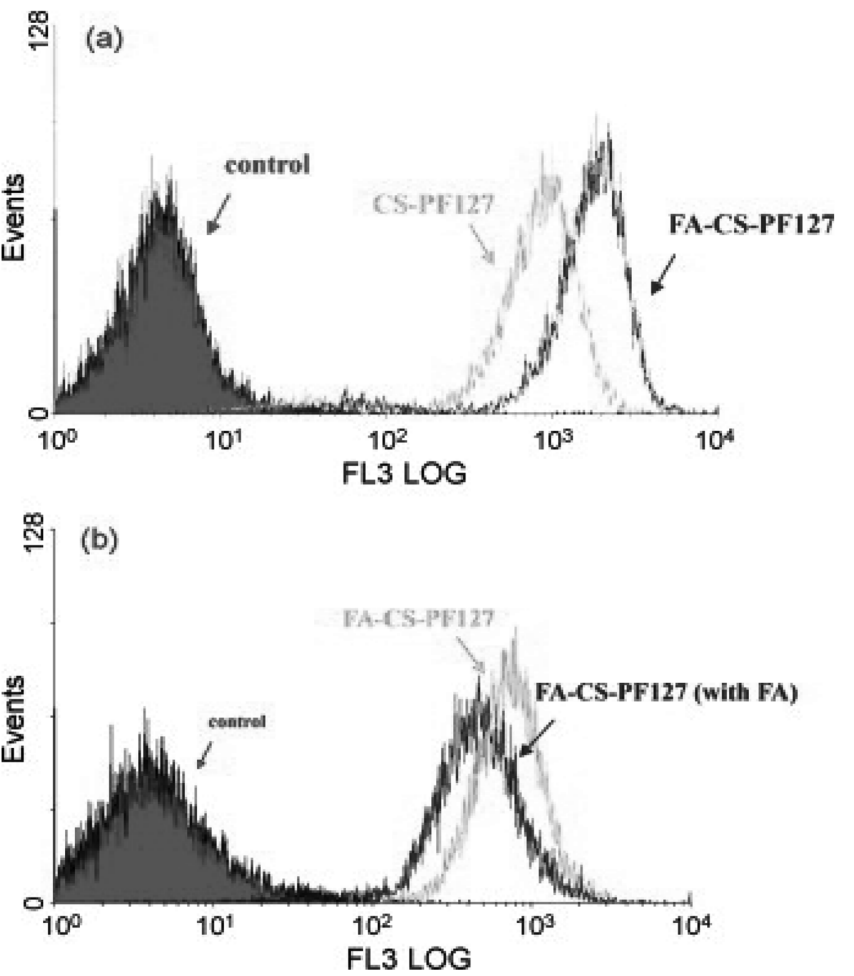

Figure 3. Flow cytometry histograms of nanogels (a), a competition study between Folic acid conjugated CS-decorated Pluronic 127 (FA-CS-PF127) and free Folic acid (FA) into KB cells (b). (reproduced from (Huang et al., 2009) with permission from the publisher) thereby restricting the cellular uptake of FA-CS-PF127 into KB cells. This result indicates that, FA-CS-PF127 has the potential of being used as a specific anti-cancer drug carrier owing to the FR-binding receptor-mediated mechanism since folic acid receptor (FR) is overexpressed in many cancerous cells. In a recent study, Bae et al. reported on self-quenching polysaccharide-based nanogels of PUL/folate-photosensitizer conjugates for photodynamic therapy (PDT) (Bae and $\mathrm{Na}$, 2010). In this study, PUL/folate-pheophorbide conjugates (PFPs) were investigated for their potential as a photosensitizer carrier (Fig. 4).

As previously mentioned, PUL/folate nanogels have a great potential as an anti-cancer drug delivery carrier (Kim et al., 2008). In this study, folate was introduced in order to endow this nanogel with a tumor-homing property by a specific interaction with folate receptors overexpressed on the surface of tumor cells. The PFP nanogels were self-organized to a greater extent than the PUL/folate conjugate during nanogel formation, due to the availability of a larger number of hydrophobic moieties as photosensitizers for formation of the hydrophobic core. Therefore, while the PFP nanogels showed photoactive properties including fluorescence and singlet oxygen generation in organic solvent (DMF), these properties were suppressed in PBS due to the self-quenching of photosensitizer moieties similar to the fluorescence resonance energy transfer (FRET) effect. From this result, it appears that self-quenching PFP nanogels may contribute in [to] the development of a new generation of photosensitizer carriers for enhanced tumor treat-
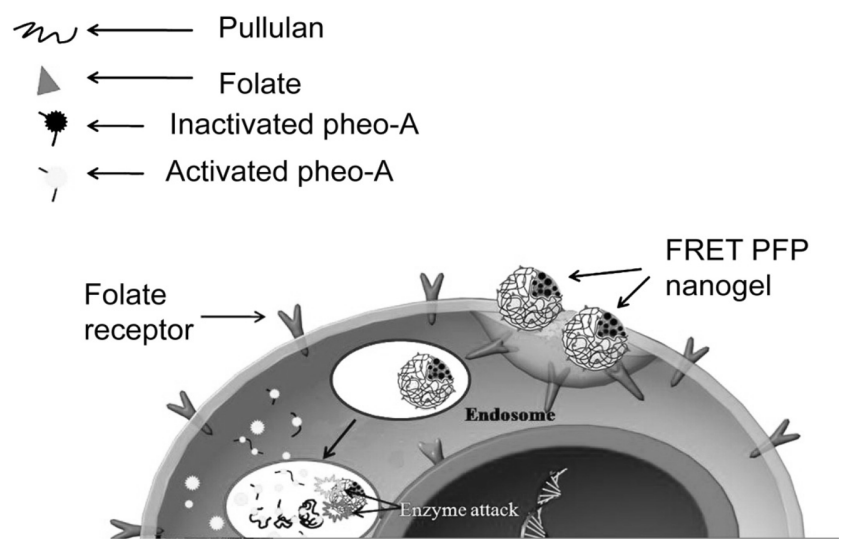

Figure 4. Conceptual image of the change in photoactivity of pullulan/folate-pheophorbide conjugates (PFP) nanogels in cells. PFP nanogels do not show photoactivity during blood circulation due to self-quenching of photosensitizers (FRET effect). When the nanogels are internalized in cancer cells, photoactivity is restored due to loss of the FRET effect by enzymatic attack within cellular compartments such as lysosomes.

(reproduced from (Bae and $\mathrm{Na}, 2010$ ) with permission from the publisher) 

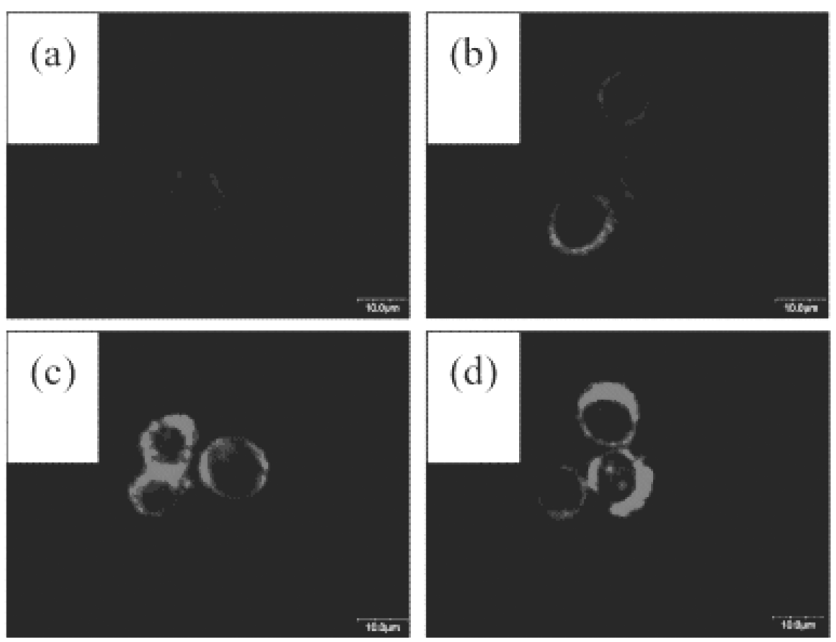

Figure 5. The different degree of internalization of Biotin conjugated pullulan acetate (BPA) nanoparticles via the endocytotic mechanism by a vitamin-receptor mediated interaction. The luminescence image of rhodamine-B-isothiocyanate (RITC) was observed by confocal microscopy. (a)Pullulan Acetate (PA), (b) BPA 1, (c) BPA 2 and (d) BPA 3.

(reproduced from(Na et al., 2003a) with permission from the publisher)

ment using PDT.

Biotin is another vitamin that has been studied as a tumor targeting ligand (Correa-Gonzalez et al., 2003; Lee et al., 2003). Biotin content in cancerous tumors is higher than that in the normal tissues and it has been found in the cell nucleus due to specific transfer of biotin to histones by human serum biotinidase (Hymes and Wolf, 1999). Na et al. reported on the self-organized nanogels of hydrophobically-modified polysaccharide bearing biotin as a targeted anti-cancer drug delivery system ( $\mathrm{Na}$ et al., 2003a). Biotin conjugated PUL acetate (BPA) exhibited high adsorption to HepG2 cells and the degree of internalization into cancer cells increased with increasing biotin content ( $\mathrm{Na}$ et al., 2003a) (Fig. 5). Yao et al. studied about preparation and characterization of biotinylated $\mathrm{ChS}$ nanoparticles (Yao et al., 2007). Biotinylated ChS (Bio-CS) was synthesized by reaction between sulfosuccinimidobiotin and ChS. They determined the difference between biotinylated ChS nanoparticles (Bio-CS-NP) and non-modified CS nanoparticles (CS-NP) against HepG2 cells.

Immunoglobulins or antibodies are a group of glycoproteins. In general, tumor cells express antigens on the surface specific to their lineage, signaling proteins, growth receptors, and in Bcell proliferative syndromes, B-cells express membrane immunoglobulins. Thus, the antibody-decorated drug-loaded nanogels can selectively target the tumor and release large amounts of a drug content into the cell cytoplasm. Currently, drug- conjugated antibodies are being designed and com- mercially distributed. More recently, Wang et al. studied the targeted herceptin-dextran iron oxide nanoparticles for noninvasive imaging of HER2/neu receptors using MRI (Chen et al., 2009).

\section{Self-organized Nanogels with Stimuli-Sensitivity}

Stimuli-sensitive polymers can respond to changes in chemical and physical conditions, such as $\mathrm{pH}$ and temperature. In a recent study, nanocarriers responsive to physical stimuli such as heat, light, sound, $\mathrm{pH}$ were studied to achieve enhanced targeted chemotherapy (Bae et al., 2005; Borisov and Zhulina, 2005; Cohn et al., 2003; Kakizawa et al., 1999; Rapoport, 2004; 2006). Among these, temperature-sensitive nanogels were first designed to achieve the purpose. There have been reports on the feasibility of thermo-sensitive nanocarriers for double-targeting of anticancer drugs to tumors, i.e., passive accumulation/aggregation and the enhanced release of loaded drugs by externally provided hyperthermic conditions (Bonifati et al., 2000; Loungnarath et al., 2005). More recently, various $\mathrm{pH}$-sensitive nanocarriers have been developed to target tumor acidity or endosomal $\mathrm{pH}$ in tumor cells (Lee et al., 2003). These nanocarriers showed fast release kinetics at tumor sites by particle disruption (Lee et al., 2003) or by cleavage of the linkage between carrier and drug (Bae et al., 2005; Nishiyama et al., 2005). This approach is expected to overcome the problems such as nonspecific toxicity, lack of tumor selectivity, and MDR in various tumor cells associated with free chemoagents. Besides $\mathrm{pH}$ and temperature sensitive nanocarriers, nanocarriers which are also sensitive simultaneously to multiple triggers have already been investigated. For example, Bhattacharya et al. engineered hybrid nanocarriers that contain ferromagnetic nanoparticles and which are responsive to both $\mathrm{pH}$, temperature, and magnetic fields (Bhattacharya et al., 2007).

\section{Temperature-Sensitive Nanogels}

The pharmaceutical relevance of thermosensitive nanocarriers lies in the treatment of local infections (with elevated environmental temperature), tumour hyperthermia or by artificially increasing temperature in the affected tissue to control local drug delivery (Ganta et al., 2008; Malmsten, 2006). Accumulation of a drug loaded nanocarrier at the disease site, followed by a increase in local temperature may trigger drug release. PNIPAAm is the most widely investigated thermoresponsive polymer for biomedical applications (Dincer et al., 2006). These thermosensitive polymers have a lower critical 
solution temperature (LCST) in aqueous solution. Although the nanocarriers are stable at low temperatures, their surface properties switch from hydrophilic to hydrophobic at elevated temperatures, resulting in nanogel aggregation, reorganization and probably enhanced interactions with cells. The switching mechanism may accelerate the drug release. Stability of the temperature-dependent nanogels arises from properties of PNIPAAm and a sharp change in the nanogel's surface hydrophilicity occurs when the temperature crosses the low critical solution temperature (LCST) of PNIPAAm. The LCST or CP of NIPAAm-based polymer can be controlled by copolymerization technique. When the nanocarrier's aggregation/reorganization temperature is tuned to around $40^{\circ} \mathrm{C}$, the nanocarrier's technology could be linked to local hyperthermic treatment of tumors at $42^{\circ} \mathrm{C}$ by combined therapy and potential synergistic effect. However, although temperature-sensitive nanocarriers based on NIPAAm showed good switching properties in response to changes in temperature, their use in clinical applications is limited because they are non-degradable. Thus, researchers have tried to develop nanocarriers that are both thermo-sensitive and biodegradable (Loh and Li, 2007; Soga et al., 2004a; Soga et al., 2004b).

Akiyoshi et al. reported on thermosensitive nanoparticles formed by self-assembly of hydrophobically modified PUL and poly(N-isopropylacrylamides) (Akiyoshi et al., 2000). Thermoresponsive hydrogel nanoparticles were prepared by self-assembly of two different hydrophobically modified polymers, namely a cholesterol-bearing PUL (CHP) and a copolymer of N-isopropylacrylamide (NIPAM) and N-[4-(1-pyrenyl)
butyl]-N-n-octadecylacrylamide] (PNIPAM-C18Py) (Fig. 6). Cholesterol-bearing PUL (CHP) form monodisperse spherical nanoparticles in water and are made up of a small number of self-aggregated CHP molecules. The PUL main chains are cross-linked via associating cholesteryl moieties. Hydrophobically modified poly(N-isopropylacrylamides) (HM-PNIPAM) also form polymeric nanogels in water, but there is quite a difference between CHP and HM-PNIPAM (Ringsdorf et al., 1992a; b; Ringsdorf et al., 1991). HM-PNIPAM nanogels consist of hydrophobic domains surrounded by hydrophilic polymer main chains. They are stable in cold water, but they are disrupted when the temperature of their solutions are increased above a critical temperature. In this study, two different hydrophobically modified polymers are mixed through association of hydrophobic groups. The hydrophobically modified polysaccharide nanoparticles have been applied in various fields. Thus, this method of using a polysaccharide mixed thermoresponsive nanoparticles is a novel application in fields such as anticancer drug delivery system, and protein delivery carrier system.

\section{pH-Sensitive Nanogels}

The extracellular $\mathrm{pH}\left(\mathrm{pH}_{\mathrm{e}}\right)$ in most tumors is more acidic $(\mathrm{pH},<7.0)$ than in normal tissues (Engin et al., 1995; Hobbs et al., 1998; Tannock and Rotin, 1989; van Sluis et al., 1999) due to the high rate of glycolysis in tumor cells under either aerobic or anaerobic conditions. The acidity mainly depends on the tumor's histology and volume. Recent measurements of $\mathrm{pH}_{\mathrm{e}}$ by noninvasive technology involving magnetic resonance spec-

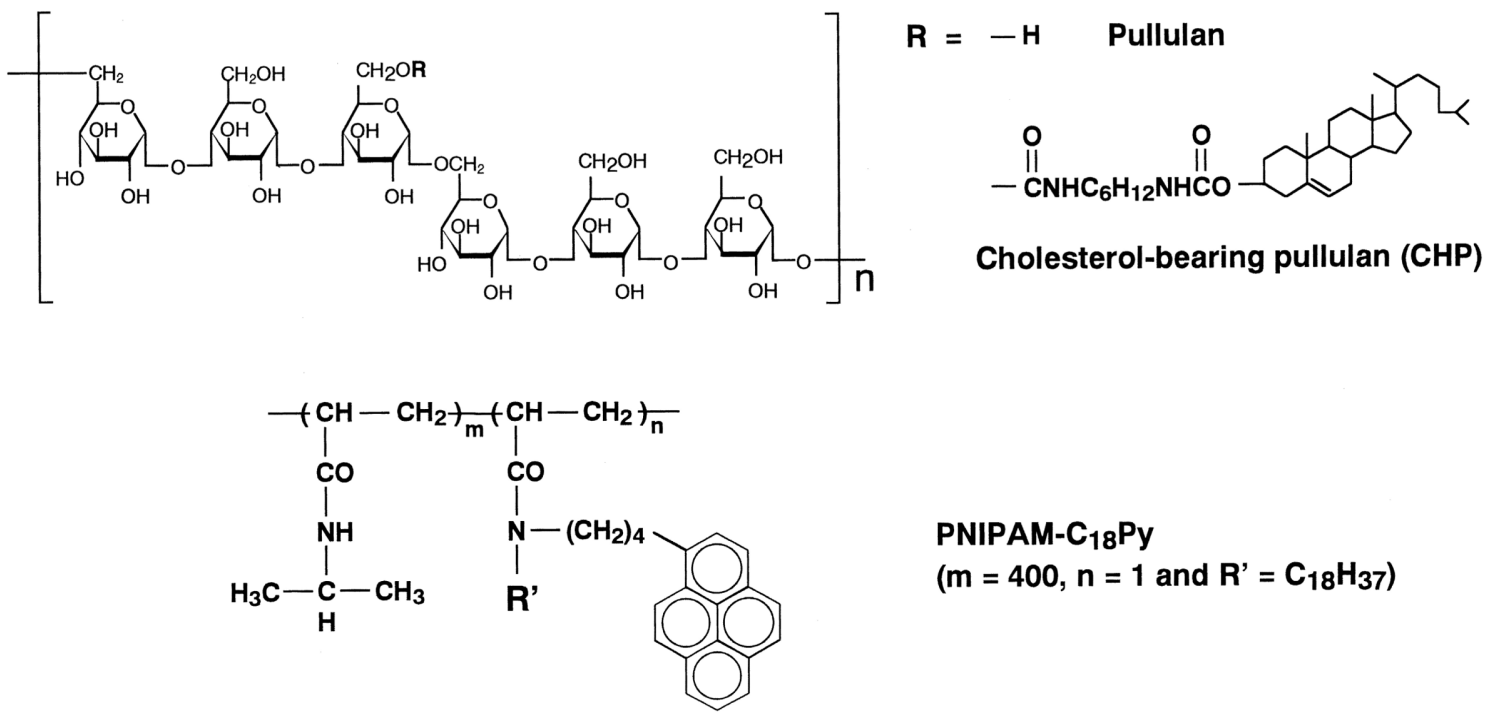

Figure 6. Chemical structure of a cholesterol-bearing pullulan (CHP) and a copolymer of N-isopropylacrylaminde (NIPAM) and N-[-4-(1pyrenyl)butyl]-N-n-octadecylacrylamide] (PNIPAM-C18Py).

(reproduced from (Akiyoshi et al., 2000) with permission from the publisher) 


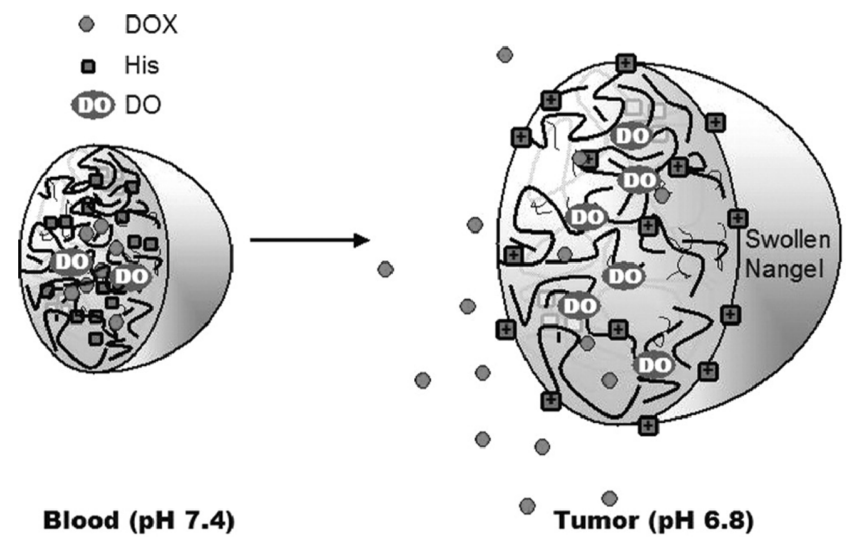

Figure 7. Schematic representation of $\mathrm{pH}$-dependent anti-cancer drug release from proposed $\mathrm{pH}$-sensitive Pullulan-Deoxycholic ac$\mathrm{id} / \mathrm{N}_{\alpha}$-Boc-L-histidine nanogels (PUL-DO/bHis). At normal $\mathrm{pH}$ (7.4), the nanogels are stable, and so the drug release rate is slower. However, at tumor acidic $\mathrm{pH}(6.8)$, the nanogels evidence swelling or disruption behavior as the result of changes in internal structure, resulting in accelerated drug release.

(reproduced from (Na et al., 2007) with permission from the publisher)

troscopy of human tumor xenografts in animals showed, and thus further confirmed a consistently low $\mathrm{pH}_{\mathrm{e}}$. All measurements (involving either invasive or noninvasive methods) of the $\mathrm{pH}_{\mathrm{e}}$ of human and animal solid tumors showed that more than $80 \%$ of all measured values are below $\mathrm{pH}$ 7.2. Several attempts at $\mathrm{pH}_{\mathrm{e}}$-triggered release using $\mathrm{pH}$-sensitive carriers have been described in recent literature (Lee et al., 2003; Na et al., 2004), but a practical option triggering the release at tumor's $\mathrm{pH}_{\mathrm{e}}$, is not even close to the desired level because of the technical difficulties in endowing the desired $\mathrm{pH}$ sensitivity to the carriers. Na et al. reported on adriamycin loaded PUL acetate/sulfonamide conjugate nanoparticles responding to tumor $\mathrm{pH}$ (Na et al., 2003b). In this study, adriamycin (ADR)loaded and $\mathrm{pH}$-sensitive nanoparticles synthesized sulfadimethoxine (SDM), was coupled to succinylated PUL Acetate (PA). In these results, depending on the intelligence properties of the $\mathrm{pH}$ sensitive nanogels, the deformation of nanogels can be reversible. In this way, nanogels can regain their original shape and structure when the stimulus triggering, the swelling or shrinking, is removed. This 'shape memory effect' can also have its implications on drug delivery (Raemdonck et al., 2009).

$\mathrm{Na}$ et al. reported on self-organized nanogels responding to tumor extracellular $\mathrm{pH}$. The self-organized nanogels were designed so that it can be stimulated by a tumor $\mathrm{pH}$ ( $\mathrm{Na}$ et al., 2007). The doxorubicin-loaded self-organized nanogels were composed of hydrophobized PUL- $\mathrm{N}_{\alpha}$-Boc-L-histidine conjugates. Deoxycholic acid (DO) was introduced as noncovalent
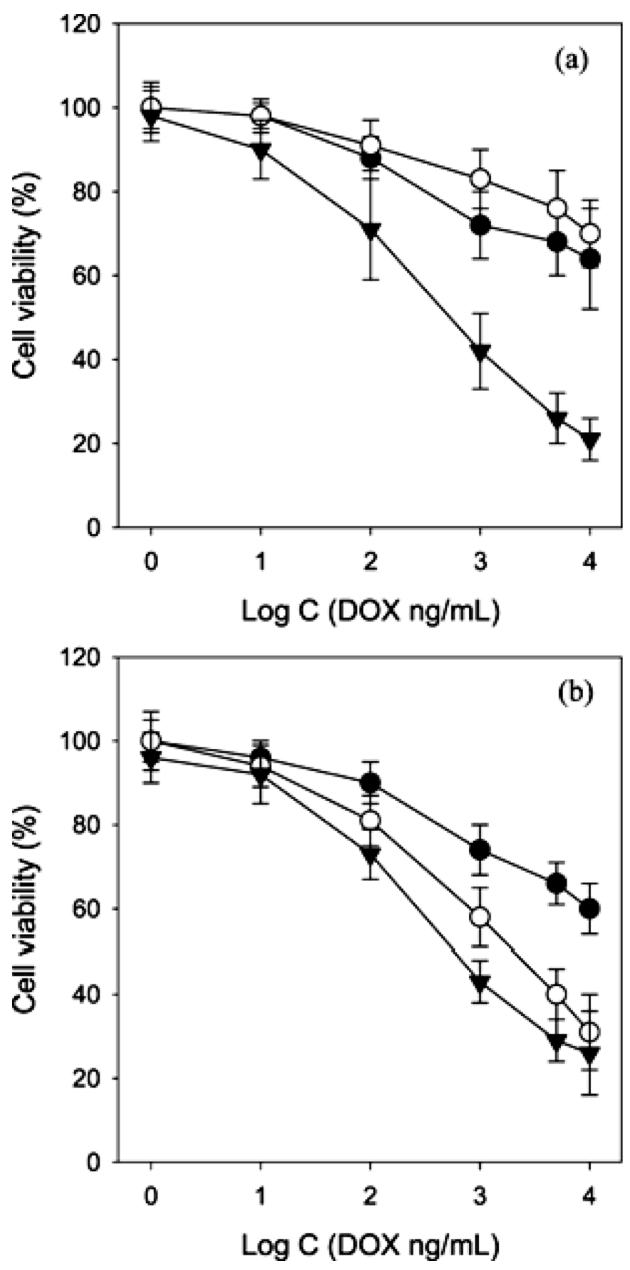

Figure 8. Cytotoxicity of Doxorubicin(DOX) loaded Pullulan- Deoxycholic acid conjugate (PUL-DO) and Pullulan- Deoxycholic acid/ $\mathrm{N}_{\alpha}$-Boc-L-histidine nanogels(PUL-DO/bHis) against MCF-7 cells, as a function of drug concentration at (a) $\mathrm{pH} 7.4$ and (b) 6.8 : $\square$ PUL-DO (•), PUL-DO/bHis78 $(\bigcirc)$, and free DOX $(\boldsymbol{\nabla})$. (reproduced from (Na et al., 2007) with permission from the publisher)

cross-linking moiety into PUL and N-Boc-histidine (bHis) was introduced as a $\mathrm{pH}$-sensitive moiety into $\mathrm{PUL} / \mathrm{DO}$ conjugate (Fig. 7). The in vitro release behaviors of DOX from DOXloaded PUL-DO/bHis at various $\mathrm{pHs}$ of $8.0,7.4,6.8$, and 6.2 were examined. The amount of DOX released increased with decreasing medium $\mathrm{pH}$ as a result of the $\mathrm{pH}$-dependent solubility of DOX, and therefore the result evidenced some slight pH-dependent DOX release behaviors. The cytotoxicity of PUL-DO/bHis nanogels on MCF-7 cells was examined at two different $\mathrm{pH}$ values of 7.4 and 6.8. At a $\mathrm{pH}$ of 7.4 , no significant difference was seen in the cytotoxicity of PUL-DO and PUL-DO/bHis nanogels. But at a $\mathrm{pH}$ of 6.8 , the DOXloaded PUL-DO/bHis nanogels evidenced significantly enhanced cytotoxicity, whereas the DOX-loaded PUL-DO nanogels evidenced no $\mathrm{pH}$ effects on cytotoxicity. The pronounced cyto- 
toxicity of PUL-DO/bHis at $\mathrm{pH}$ of 6.8 may be attributed to the enhanced DOX release rate. So, they suggested a potential method in order to deliver anticancer drugs effectively by using polyHis-based nanogels, and thus, His-based nanocarriers have received a great deal of attention as an anti-tumor drug carrier (Fig. 8).

\section{Conclusions}

As reviewed above, numerous polysaccharide based nanogels for tumor targeted drug delivery have been developed. These nanogels are generally investigated in terms of their physicochemical properties, drug loading ability, in vitro and in vivo toxicity tests. The most important property of nanogels for tumor targeting is the tumor specific interaction. High interaction of a drug carrier with the target tumor cells is ideal for tumor chemotherapy. For this purpose, many endogenous and exogenous ligands or ligand-carrier derivates have been utilized for active targeting to various specific and non-specific cellular mechanisms. Most of these approaches are based on cell-surface receptors or cell-surface epitopes that mediate trafficking of ligands to subcellular organelles or to the cytosol. Among the ligands, vitamins have been employed and have shown higher internalization rate or binding to cancer cells. However, the specificity of a ligand to a tumor is only for 'active internalization' into the specific tumor with minimal interactions with other tissues. Stimuli-sensitive nanogels were also investigated for tumor targeting of anticancer drugs. The development of various stimuli-sensitive nanogels prompted the creation of a new mode of anticancer drug delivery systems. The system allows for the minimization of problems such as nonspecific toxicity, lack of tumor selectivity, and development of MDR in various tumor cells, associated with chemoagents. Drug release triggered by external signals or pathophysiological conditions at tumor sites is expected to enhance tumor specific chemotherapy.

\section{Acknowledgments}

This work was financially supported by Grant No.A084060 from the Korean Ministry for Health Welfare and Family Affairs funds.

\section{References}

Akiyoshi, K., Kang, E.C., Kurumada, S., Sunamoto, J., Principi, T., Winnik, F.M., 2000. Controlled association of amphiphilic polymers in water: thermosensitive nanoparticles formed by self-assembly of hydrophobically modified pullulans and poly (N-isopropylacrylamides). Macromolecules 33, 3244-3249.

Aruffo, A., Stamenkovic, I., Melnick, M., Underhill, C.B., Seed, B., 1990. CD44 is the principal cell surface receptor for hyaluronate. Cell 61, 1303-1313.

Bae, B.C., Na, K., 2010. Self-quenching polysaccharide-based nanogels of pullulan/folate-photosensitizer conjugates for photodynamic therapy. Biomaterials 31, 6325-6335.

Bae, Y., Kataoka, K., 2006. Significant enhancement of antitumor activity and bioavailability of intracellular $\mathrm{pH}$-sensitive polymeric micelles by folate conjugation. J. Controlled Release 116, e49-50.

Bae, Y.S., Nishiyama, N., Fukushima, S., Koyama, H., Yasuhiro, M., Kataoka, K., 2005. Preparation and biological characterization of polymeric micelle drug carriers with intracellular $\mathrm{pH}$-triggered drug release property: tumor permeability, controlled subcellular drug distribution, and enhanced in vivo antitumor efficacy. Bioconjugate Chem 16, 122-130.

Bagalkot, V., Farokhzad, O.C., Langer, R., Jon, S., 2006. An aptamer-doxorubicin physical conjugate as a novel targeted drug-delivery platform. Angew. Chem. 118, 8329-8332.

Balthasar, S., Michaelis, K., Dinauer, N., von Briesen, H., Kreuter, J., Langer, K., 2005. Preparation and characterisation of antibody modified gelatin nanoparticles as drug carrier system for uptake in lymphocytes. Biomaterials 26, 2723-2732.

Banerji, S., Ni, J., Wang, S.X., Clasper, S., Su, J., Tammi, R., Jones, M., Jackson, D.G., 1999. LYVE-1, a new homologue of the CD44 glycoprotein, is a lymph-specific receptor for hyaluronan. The Journal of cell biology 144, 789.

Benhar, I., Padlan, E., Jung, S., Lee, B., Pastan, I., 1994. Rapid humanization of the Fv of monoclonal antibody B3 by using framework exchange of the recombinant immunotoxin B3 (Fv)-PE38. Proc. Natl. Acad. SCI. USA. 91, 12051.

Bhattacharya, S., Eckert, F., Boyko, V., Pich, A., 2007. Temperature-, $\mathrm{pH}-$, and magnetic-field-sensitive hybrid microgels. Small 3, 650-657.

Bonifati, D., Ori, C., Rossi, C., Caira, S., Fanin, M., Angelini, C., 2000. Neuromuscular damage after hyperthermic isolated limb perfusion in patients with melanoma or sarcoma treated with chemotherapeutic agents. Cancer Chemother. Pharmacol. 46, 517-522.

Borisov, O.V., Zhulina, E.B., 2005. Reentrant morphological transitions in copolymer micelles with $\mathrm{pH}$-sensitive corona. Langmuir 21, 3229-3231.

Bougrine, R., Masson, C., Hatier, R., Nex , E., Nicolas, J., Gueant, J., 1996. Receptor binding of transcobalamin II-cobalamin in human colon adenocarcinoma HT 29 cell line. J. Nutr. Biochem. 7, 397-402.

Brigger, I., Dubernet, C., Couvreur, P., 2002. Nanoparticles in cancer therapy and diagnosis. Adv. Drug Del. Rev. 54, 631-651.

Chen, T.J, Cheng, T.H., Chen, C.Y., Hsu, S., Cheng, T.L., Liu, G.C., Wang, Y.M., 2009. Targeted herceptin-dextran iron oxide nanoparticles for noninvasive imaging of HER2/neu receptors using MRI. J. Biol. Inorg. Chem. 14, 253-260. 
Choi, K.Y., Chung, H., Min, K.H., Yoon, H.Y., Kim, K., Park, J.H., Kwon, I.C., Jeong, S.Y., 2010, Self-ssembled hyaluronic acid nanoparticles for active tumor targeting. Biomaterials 31, 106114.

Cohn, D., Sosnik, A., Levy, A., 2003. Improved reverse thermoresponsive polymeric systems. Biomaterials 24, 3707-3714.

Collins, D.A., Hogenkamp, H.D., O'Connor, M.K., Naylor, S., Benson, L.M., Hardyman, T.J., Thorson, L.M., 2000. Biodistribution of radiolabeled adenosylcobalamin in patients diagnosed with various malignancies. Mayo Clinic, 568-580.

Correa-Gonzalez, L., Arteaga de Murphy, C., Ferro-Flores, G., Pedraza-Lopez, M., Murphy-Stack, E., Mino-Leon, D., PerezVillasenor, G., Diaz-Torres, Y., Munoz-Olvera, R., 2003. Uptake of 153Sm-DTPA-bis-biotin and 99mTc-DTPA-bisbiotin in rat as-30D-hepatoma cells. Nucl. Med. Biol. 30, 135140.

Dincer, S., Rzaev, Z.M.O., Piskin, E., 2006. Synthesis and Characterization of Stimuli-Responsive Poly (N-isopropylacrylamide-co-N-vinyl-2-pyrrolidone). J. Polym. Res. 13, 121-131.

Engin, K., Leeper, D.B., Cater, J.R., Thistlethwaite, A.J., Tupchong, L., McFarlane, J.D., 1995. Extracellular pH distribution in human tumours. IJHy 11, 211-216.

Flodh, H., Ullberg, S., 1968. Accumulation of labelled vitamin B12 in some transplanted tumours. Int. J. Cancer 3, 694-699.

Ganta, S., Devalapally, H., Shahiwala, A., Amiji, M., 2008. A review of stimuli-responsive nanocarriers for drug and gene delivery. J. Controlled Release 126, 187-204.

Henke, C.A., Roongta, U., Mickelson, D.J., Knutson, J.R., McCarthy, J.B., 1996. CD44-related chondroitin sulfate proteoglycan, a cell surface receptor implicated with tumor cell invasion, mediates endothelial cell migration on fibrinogen and invasion into a fibrin matrix. J. Clin. Invest. 97, 2541-2552.

Hobbs, S.K., Monsky, W.L., Yuan, F., Roberts, W.G., Griffith, L., Torchilin, V.P., Jain, R.K., 1998. Regulation of transport pathways in tumor vessels: role of tumor type and microenvironment. Proc. Natl. Acad. Sci. U. S. A. 95, 4607-4612.

Homma, A., Sato, H., Tamura, T., Okamachi, A., Emura, T., Ishizawa, T., Kato, T., Matsuura, T., Sato, S., Higuchi, Y., 2009. Synthesis and optimization of hyaluronic acid-methotrexate conjugates to maximize benefit in the treatment of osteoarthritis. Biorg. Med. Chem.,1062-1075

Huang, S.H., Sun, S.L., Feng, T.H., Sung, K.H., Lui, W.L., Wang, L.F., 2009. Folate-mediated chondroitin sulfate-Pluronic ${ }^{\circledR} 127$ nanogels as a drug carrier. Eur. J. Pharm. Sci. 38, 64-73.

Hwang, H.Y., Kim, I.S., Kwon, I.C., Kim, Y.H., 2008. Tumor targetability and antitumor effect of docetaxel-loaded hydrophobically modified glycol chitosan nanoparticles. J. Controlled Release 128, 23-31.

Hymes, J., Wolf, B., 1999. Human biotinidase isn't just for recycling biotin. J. Nutr. 129, 485-489.

Jaracz, S., Chen, J., Kuznetsova, L.V., Ojima, I., 2005. Recent advances in tumor-targeting anticancer drug conjugates. Biorg. Med. Chem. 13, 5043-5054.

Jeong, Y.I, Na, H.S., Oh, J.S., Choi, K.C., Song, C.E., Lee, H.C.,
2006. Adriamycin release from self-assembling nanospheres of poly (dl-lactide-co-glycolide)-grafted pullulan. Int. J. Pharm. 322, 154-160.

Jeong, Y.I., Nah, J.W., Na, H.K., Na, K., Kim, I.S., Cho, C.S., Kim, S.H., 1999. Self-assembling nanospheres of hydrophobized pullulans in water. Drug Dev. Ind. Pharm. 25, 917-927.

Jung, S.W., Jeong, Y.I., Kim, S.H., 2003. Characterization of hydrophobized pullulan with various hydrophobicities. Int. J. Pharm. 254, 109-121.

Kabanov, A.V., Vinogradov, S.V., 2009. Nanogels as pharmaceutical carriers: finite networks of infinite capabilities. Angew. Chem. Int. Edit. 48, 5418-5429.

Kakizawa, Y., Harada, A., Kataoka, K., 1999. Environment-sensitive stabilization of core- shell structured polyion complex micelle by reversible cross-linking of the core through disulfide bond. J. Am. Chem. Soc 121, 11247-11248.

Kaneo, Y., Tanaka, T., Nakano, T., Yamaguchi, Y., 2001. Evidence for receptor-mediated hepatic uptake of pullulan in rats. $\mathrm{J}$. Controlled Release 70, 365-373.

Khaw, B.A., Klibanov, A., O'Donnell, S.M., Saito, T., Nossiff, N., Slinkin, M.A., Newell, J.B., Strauss, H.W., Torchilin, V.D., 1991. Gamma imaging with negatively charge-modified monoclonal antibody: modification with synthetic polymers. J. Nucl. Med. 32, 1742.

Kim, S., Park, K.M., Ko, J.Y., Kwon, I.C., Cho, H.G., Kang, D., Yu, I.T., Kim, K., Na, K., 2008. Minimalism in fabrication of self-organized nanogels holding both anti-cancer drug and targeting moiety. Colloids Surf. B. Biointerfaces 63, 55-63.

Kumar, M.N., Muzzarelli, R.A., Muzzarelli, C., Sashiwa, H., Domb, A.J., 2004. Chitosan chemistry and pharmaceutical perspectives. Chem. Rev. 104, 6017-6084.

Laurent, T.C., 1998. The chemistry, biology and medical applications of hyaluronan and its derivatives. Portland Pr .London, U.K.

Leathers, T.D., 2003. Biotechnological production and applications of pullulan. Appl. Microbiol. Biotechnol. 62, 468-473.

Lee, E.S., Na, K., Bae, Y.H., 2003. Polymeric micelle for tumor $\mathrm{pH}$ and folate-mediated targeting. J. Control Release 91, 103-113.

Letchford, K., Burt, H., 2007. A review of the formation and classification of amphiphilic block copolymer nanoparticulate structures: micelles, nanospheres, nanocapsules and polymersomes. Eur. J. Pharm. Biopharm. 65, 259-269.

Liu, Z., Jiao, Y., Wang, Y., Zhou, C., Zhang, Z., 2008. Polysaccharides-based nanoparticles as drug delivery systems. Adv. Drug Del. Rev. 60, 1650-1662.

Loh, X.J., Li, J., 2007. Biodegradable thermosensitive copolymer hydrogels for drug delivery. Expert. Opin. Thera. Pat. 17, 965977.

Loungnarath, R., Causeret, S., Bossard, N., Faheez, M., SayagBeaujard, A.C., Brigand, C., Gilly, F., Glehen, O., 2005. Cytoreductive surgery with intraperitoneal chemohyperthermia for the treatment of pseudomyxoma peritonei: a prospective study. Dis. Colon Rectum 48, 1372-1379.

Lu, Y., Low, P.S., 2002. Folate-mediated delivery of macromo- 
lecular anticancer therapeutic agents. Adv. Drug Del. Rev. 54, 675-693.

Lugli, A., Zlobec, I., Gunthert, U., Minoo, P., Baker, K., Tornillo, L., Terracciano, L., Jass, J., 2006. Overexpression of the receptor for hyaluronic acid mediated motility is an independent adverse prognostic factor in colorectal cancer. Mod. Pathol. 19, 1302-1309.

Luo, Y., Prestwich, G.D., 1999. Synthesis and Selective Cytotoxicity of a Hyaluronic Acid- Antitumor Bioconjugate. Bioconjugate Chem. 10, 755-763.

Luo, Y., Ziebell, M., Prestwich, G., 2000. A Hyaluronic AcidTaxol Antitumor Bioconjugate Targeted to Cancer Cells. Biomacromolecules 1, 208-218.

Malmsten, M., 2006. Soft drug delivery systems. Soft Matter 2, 760-769.

Mohanraj, V.J., Chen, Y., 2007. Nanoparticles-A review. Trop. J. Pharm. Res. 5, 561-573.

Mucci, A., Schenetti, L., Volpi, N., 2000. 1H and 13C nuclear magnetic resonance identification and characterization of components of chondroitin sulfates of various origin. Carbohydr. Polym. 41, 37-45.

Na, K., Bum Lee, T., Park, K.H., Shin, E.K., Lee, Y.B., Choi, H.K., 2003a. Self-assembled nanoparticles of hydrophobically-modified polysaccharide bearing vitamin $\mathrm{H}$ as a targeted anti-cancer drug delivery system. Eur. J. Pharm. Sci. 18, 165-173.

Na, K., Lee, E.S., Bae, Y.H., 2007. Self-organized nanogels responding to tumor extracellular $\mathrm{pH}$ : $\mathrm{pH}$-dependent drug release and in vitro cytotoxicity against MCF-7 cells. Bioconjugate Chem 18, 1568-1574.

Na, K., Lee, K.H., Bae, Y.H., 2004. pH-sensitivity and pH-dependent interior structural change of self-assembled hydrogel nanoparticles of pullulan acetate/oligo-sulfonamide conjugate. J. Controlled Release 97, 513-525.

Na, K., Park, K.M., Jo, E.A., Lee, K.S., 2006. Self-organized pullulan/deoxycholic acid nanogels: Physicochemical characterization and anti-cancer drug-releasing behavior. Biotechnol. Bioproc. E 11, 262-267.

Na, K., Seong Lee, E.S., Bae, Y.H., 2003b. Adriamycin loaded pullulan acetate/sulfonamide conjugate nanoparticles responding to tumor $\mathrm{pH}$ : $\mathrm{pH}$-dependent cell interaction, internalization and cytotoxicity in vitro. J. Controlled Release 87, 3-13.

Nishiyama, N., Bae, Y., Miyata, K., Fukushima, S., Kataoka, K., 2005. Smart polymeric micelles for gene and drug delivery. Drug. Discov. Today 2, 21-26.

Nishiyama, N., Kataoka, K., 2006. Current state, achievements, and future prospects of polymeric micelles as nanocarriers for drug and gene delivery. Pharmacol. Ther. 112, 630-648.

Nobs, L., Buchegger, F., Gurny, R., Allemann, E., 2004. Current methods for attaching targeting ligands to liposomes and nanoparticles. J. Pharm. Sci. 93, 1980-1992.

Normanno, N., Bianco, C., Strizzi, L., Mancino, M.R., Maiello, M., Luca, A., Caponigro, F., Salomon, D.S., 2005. The ErbB receptors and their ligands in cancer: an overview. Curr. Drug Targets 6, 243-257.
Oh, J.K., Drumright, R., Siegwart, D.J., Matyjaszewski, K., 2008. The development of microgels/nanogels for drug delivery applications. Prog. Polym. Sci. 33, 448-477.

Park, K., Kim, J.H., Nam, Y.S., Lee, S., Nam, H.Y., Kim, K., Park, J.H., Kim, I.S., Choi, K., Kim, S.Y. Kwon I.C., 2007. Effect of polymer molecular weight on the tumor targeting characteristics of self-assembled glycol chitosan nanoparticles. J. Controlled Release 122, 305-314.

Park, W., Kim, K.S., Bae, B.C., Kim, Y.H., Na, K., 2010. Cancer cell specific targeting of nanogels from acetylated hyaluronic acid with low molecular weight. Eur. J. Pharm. Sci. 40, 367375.

Park, W., Park, S.J., Na, K.,2010. Potential of self-organizing nanogel with acetylated chondroitin sulfate as an anti-cancer drug carrier. Colloids Surf. B. Biointerfaces. 501-508.

Raemdonck, K., Demeester, J., Smedt, S.D., 2009. Advanced nanogel engineering for drug delivery. Soft Matter 5, 707-715.

Rapoport, N., 2004. Combined cancer therapy by micellar-encapsulated drug and ultrasound. Int. J. Pharm. 277, 155-162.

Rapoport, N., 2006. Tumor Targeting by Polymer Assemblies and Ultrasound Activation. MML series 8, 305-362.

Rapoport, N., 2007. Physical stimuli-responsive polymeric micelles for anti-cancer drug delivery. Prog. Polym. Sci. 32, 962-990.

Rekha, M.R., Sharma, C.P., 2007. Pullulan as a promising biomaterial for biomedical applications: A perspective. Trends Biomater. artif. Organs. 20, 116-121.

RIcKms, E., Brink, N., lactis Dorner, L., 1948. Crystalline vitamin B12. Sci 107. 396-397

Ringsdorf, H., Simon, J., Winnik, F.M., 1992a. Hydrophobicallymodified poly ( $\mathrm{N}$-isopropylacrylamides) in water: probing of the microdomain composition by nonradiative energy transfer. Macromolecules 25, 5353-5361.

Ringsdorf, H., Simon, J., Winnik, F.M., 1992b. Hydrophobically modified poly ( $\mathrm{N}$-isopropylacrylamides) in water: a look by fluorescence techniques at the heat-induced phase transition. Macromolecules 25, 7306-7312.

Ringsdorf, H., Venzmer, J., Winnik, F.M., 1991. Fluorescence studies of hydrophobically modified poly ( $\mathrm{N}$-isopropylacrylamides). Macromolecules 24, 1678-1686.

Seetharam, B., Bose, S., Li, N., 1999. Cellular import of cobalamin (Vitamin B-12). J. Nutr. 129, 1761.

Seetharam, B., Li, N., 2000. Transcobalamin II and its cell surface receptor. Vitam. Horm. 59, 337-366.

Shen, Y., Tang, H., Radosz, M., Van Kirk, E., Murdoch, W.J, 2008. $\mathrm{pH}$-responsive nanoparticles for cancer drug delivery. Method. Mol. Biol. 437, 183.

Soga, O., van Nostrum, C.F., Hennink, W.E., 2004a. Poly (N-(2hydroxypropyl) methacrylamide mono/di lactate): a new class of biodegradable polymers with tuneable thermosensitivity. Biomacromolecules 5, 818-821.

Soga, O., van Nostrum, C.F., Ramzi, A., Visser, T., Soulimani, F., Frederik, P.M, Bomans, P., Hennink, W.E., 2004b. Physicochemical characterization of degradable thermosensitive polymeric micelles. Langmuir 20, 9388-9395.

J. Pharm. Invest., Vol. 40, No. 4 (2010) 
Tanaka, T., Fujishima, Y., Hanano, S., Kaneo, Y., 2004. Intracellular disposition of polysaccharides in rat liver parenchymal and nonparenchymal cells. Int. J. Pharm. 286, 9-17.

Tannock, I.F., Rotin, D., 1989. Acid $\mathrm{pH}$ in tumors and its potential for therapeutic exploitation. Cancer Res. 49, 4373-4384.

Torchilin, V.P., 2006. Multifunctional nanocarriers. Adv. Drug Del. Rev. 58, 1532-1555.

van Sluis, R., Bhujwalla, Z.M., Raghunand, N., Ballesteros, P., Alvarez, J., Cerdan, S., Galons, J.P., Gillies, R.J., 1999. In vivo imaging of extracellular $\mathrm{pH}$ using $1 \mathrm{H}$ MRSI. Magn. Reson. Med. 41, 743-750.

Ward, P.D., Thibeault, S.L., Gray, S.D., 2002. Hyaluronic Acid:: Its Role in Voice. J. Voice 16, 303-309.

Wong, J.Y., Kuhl, T.L., Israelachvili, J.N., Mullah, N., Zalipsky, S., 1997. Direct measurement of a tethered ligand-receptor interaction potential. Sci $275,820-822$.
Xi, K., Tabata, Y., Uno, K., Yoshimoto, M., Kishida, T., Sokawa, Y., Ikada, Y., 1996. Liver targeting of interferon through pullulan conjugation. Pharm. Res. 13, 1846-1850.

Xin, D., Wang, Y., Xiang, J., 2010. The Use of Amino Acid Linkers in the Conjugation of Paclitaxel with Hyaluronic Acid as Drug Delivery System: Synthesis, Self-Assembled Property, Drug Release, and In Vitro Efficiency. Pharm. Res. 27, 380389.

Yang, W.Z., Zhang, Q.Q., Chen, H.L., Li, X.M., Jiang, Q., Chen, M.M, Gao, F.P., Zhang, H.Z, 2008. Preparation and physicochemical characteristics of self-assembled nanoparticles of cholesterol succinate modified pullulan conjugates. Springer, 13-17.

Yao, Q., Hou, S.X, Zhang, X., Zhao, G., Gou, X.J., You, J.Z, 2007. Preparation and characterization of biotinylated chitosan nanoparticles. A.P.S 42, 557. 